\title{
BILINGUAL GEPRÄGTE KOMMUNIKATIONSSTRUKTUREN AUF DER GRUNDLAGE FRÄNKISCHBASIERTER UNGARNDEUTSCHER MUNDARTEN DER GEGENWART
}

\author{
wammer deitsch o:fange \\ owwa ungarisch wirds ö \\ gehts aus
}

(,Wenn wir Deutsch anfangen, aber Ungarisch wird es äh, geht es aus“". Ausspruch einer 80-jährigen Informantin aus dem primär rheinfränkischen Wieland/Villány, UZSK_E_0075, S. 14)

\section{HINTERGRUND, VERORTUNG UND ZIELE}

Die konzepttheoretische und empirische Grundlage des vorliegenden Aufsatzes bildet ein aktuelles Forschungs- und Dokumentationsprojekt ${ }^{1}$ unter dem Titel „Digitales Portal ,Ungarndeutsches Zweisprachigkeits- und Sprachkontaktkorpus““. Das Vorhaben wurde von der deutschen Kulturstaatsministerin in ihrer Eigenschaft als Beauftragte der Bundesregierung für Kultur und Medien (BKM) insgesamt für einen dreieinhalbjährigen Zeitraum - erste Projektphase: vom 01.09.2015 bis 31.12.2017, zweite Projektphase vom 01.01.2018 bis 31.03.2019 - am Lehrstuhl für Germanistische Sprachwissenschaft der Universität Erfurt bewilligt (Projektnummern: K 44-41000/1\#92 bzw. K 44-41000/1\#75). ${ }^{2}$

Das Projekt ist inhaltlich zum einen im Rahmen der diatopisch orientierten Variationslinguistik (Stichwort Regionalsprachen- bzw. Sprachdynamikforschung), zum anderen in dem der Zwei- bzw. Mehrsprachigkeitsforschung und der synchron ausgerichteten Kontaktlinguistik anzusiedeln und operiert mit einer triangulativen Forschungsstrategie, siehe Földes (2016a: 322f.). Hinzu kommt die Korpuslinguistik für die Dokumentation und Erschließung. Das Hauptanliegen ist eine variations- und kontaktlinguistische Erforschung ungarndeutscher mündlicher Diskurse im interaktiven Alltag der Verständigung sowie die Erstellung eines digitalen Portals mit einem

csaba.foeldes@uni-erfurt.de; foeldes@foeldes.eu

1 Dessen Grundidee, Konzept und inhaltliche Strukturen, einschließlich auch der logistischoperativen Arbeitsschritte und -verfahren, bildeten bereits den Gegenstand mehrerer Publikationen, z. B. Földes (2016a) und (2016b), sodass sie im vorliegenden Beitrag nur knapp erörtert werden.

2 Für die Förderung sei auch an dieser Stelle vielmals gedankt. 
ungarndeutschen Zweisprachigkeits- und Sprachkontaktkorpus. Im Einzelnen geht es um die Aufzeichnung von bestandsgefährdetem regionalem Sprachmaterial des Deutschen als Minderheitensprache in Ungarn. Dies realisiert sich in der Erarbeitung eines Zweisprachigkeits- und Sprachkontaktkorpus mit echten Diskursrealisationen ${ }^{3}$ aus drei wichtigen Siedlungsregionen deutscher Minderheiten, das den zentralen Projektgegenstand bildet. Dazu wurde ein öffentlich zugängliches digitales Portal eingerichtet: Strukturiertes und kommentiertes ungarndeutsches Sprachmaterial wird vor allem für sprach- und kulturwissenschaftliche, aber auch für sozial-, mentalitäts- und alltagshistorische Studien dargeboten. Damit werden Prozesse und Folgen des sprachlich-kulturellen Austausches sowie Verflechtungen der sprachlich-kommunikativen Verhältnisse systematisch erfasst und dokumentiert (Földes 2016a: 323). ${ }^{4}$

Dieser Beitrag soll vor dem Hintergrund der obigen kurzen Skizzierung des Projektdesigns ausgewählte, aus dem Projektfundus hervorgegangene erste Forschungsbeobachtungen und -ergebnisse über einige rezente ungarndeutsche Kontaktvarietäten fränkischer Provenienz aus Südungarn (aus der sog. „Schwäbischen Türkei“) präsentieren. Ziel ist es, die aktuelle Diskursrealität an diesem Material evidenzbasiert zu erschlieBen und daraus charakteristische Prozesse, Muster und Resultate vor allem von Kontakt- bzw. Interaktionsgeschehen, die das Profil der ungarndeutschen Gegenwartsdialekte heute markant prägen, zu extrahieren. Dabei verdienen auch Effekte sukzessiver Spracherosion besondere Beachtung. Somit soll letztlich ein sprachwirklichkeitsnahes Bild der heutigen ungarndeutschen Kommunikationskultur und Kompetenzstruktur im gegebenen Kontext erarbeitet werden.

\section{DER SPRACHKOMMUNIKATIVE DISPOSITIONSRAHMEN}

Die sprachlich-kommunikative Situation der deutschen Minderheit in Ungarn zeichnet sich durch einen umfassenden und durchdringenden soziokulturellen sowie sprachlichen Austausch aus. In dieser kulturellen Fugenposition ist das Deutsche weder Mutter- noch Fremdsprache im herkömmlichen Sinne des Wortes. Das kommunikative Milieu der Ungarndeutschen wird seit über 250 Jahren durch immer intensiver werdende Außenkontakte (und z. T. Konflikte) mit dem Ungarischen - und in geringeren Ausmaß mit anderen Umgebungssprachen bzw. -varietäten - geprägt: Ungarisch übt seit der zweiten Hälfte des 19. Jahrhunderts einen sukzessiv wachsenden Druck auf das kommunikative Handeln und auf das Sprachrepertoire der Ungarndeutschen aus; der Einfluss des Ungarischen nahm schließlich nach 1945 einen übermächtigen Umfang an.

Mithin ist die soziale Wirklichkeit ,Deutsch als Minderheitensprache' heute (in der Untersuchungsregion wie auch generell) paradigmatisch durch eine markante, zugleich aber immer instabiler werdende Zwei- bzw. Mehrsprachigkeit (im Weiteren:

3 Terminus im Sinne von Roth (2015: 37f.).

4 Zum internationalen forschungsstrategischen Kontext vgl. die Feststellung von Adamou (2019: 640): „Spoken corpora are of special interest to linguists focusing on contact, as they potentially include a wide range of contact phenomena and may reveal a information about the cognitive processes involved in production and comprehension of bilingual speech“. 



gleichzeitig durch eine enge Verquickung mit dem Ungarischen als überaus dominanter Kommunikationssprache gekennzeichnet. In den verschiedenen Interaktionssituationen wird produktiv wie rezeptiv im Wesentlichen - wenn auch in unterschiedlichen Domänen und mit unterschiedlicher Häufigkeit - auf drei sprachliche Kodes und ihre subtilen Übergangs- bzw. Mischformen zurückgegriffen, und zwar auf die jeweilige ungarndeutsche Siedlungsmundart, auf die ungarische Standardvarietät und (zumindest sporadisch) auf die deutsche Standardvarietät. Das heißt, dass eine Mehrsprachigkeit bei strukturell unähnlichen Sprach(varietät)en mit ungleichwertigem Status und Prestige besteht. Dies führt zu einem asymmetrischen Charakter des Sprachkontaktes. Diese Konstellation könnte man etwa bilinguale Dialekt-Standard-Diglossie' nennen. Den in der mündlichen Ingroup-Kommunikation verwendeten besonderen, bilingual-transkulturell geprägten Varietätentyp bezeichne ich als ,Kontaktdeutsch“ (vgl. Földes 2005: 37). Dabei sind die sprachlichen Formen und ihre Diskursrealisationen durch eine außerordentlich hohe Dynamik gekennzeichnet, mitunter zeigen sich sogar Ansätze von Fluktuation sowie u. U. eine zunehmende Labilität. Folglich ist Okkasionalität ein immanentes Merkmal der gegenwärtigen ungarndeutschen Redeweise. Demzufolge praktizieren Ungarndeutsche einen spezifischen, ausgesprochen kontextgebundenen bilingual-oszillierenden Sprech- bzw. Gesprächsstil, der in Abhängigkeit von den kommunikativen Bedingungen variiert und der sogar für die Symbolisierung sozialer Identität (und Alterität) eine Rolle spielt (siehe Földes 2013: 122). In Bezug auf die Beherrschung der deutschen Sprache, in welcher Varietät auch immer, lässt sich zwischen den Extremen „sehr kompetent" und „erodierte (produktive) Kompetenz“ ein weites Kontinuum aufspannen, in dessen Mitte sich die sog. semispeaker (also Halbsprecher) ${ }^{7}$ befinden, die über eine durchaus limitierte produktive Kommunikationskompetenz verfügen. Ein nicht unwesentlicher Teil der Gewährspersonen der vorliegenden Studie gehört in diese Gruppe.

Zu den Prämissen gehört mithin: Das sprachlich-kommunikative Verhalten ungarndeutscher Sprecher, die in mehrsprachigen und polykulturellen Räumen aufwachsen und leben, unterscheidet sich grundlegend von dem einsprachiger Sprecher des Deutschen z. B. im zusammenhängenden deutschen Sprachraum. Als Reflex auf die veränderten soziokulturellen Rahmenbedingungen ist die Herausbildung neuer kommunikativer und sprachlicher Formationen selbstverständlich. So entstanden u. a. spezifische Zwischenformen und Verbindungen aus den verfügbaren Kodes, bei denen prototypisch drei Phänomenklassen in Erscheinung treten: (a) Prozesse interlingualer Transfers/Übernahmen, (b) zwischensprachliche Kopien und (c) Sprachalternierungen. Die ersten beiden Manifestationsarten fasse ich (siehe Földes 2013: 122) unter Hybridität zusammen, während Typ (c) als Synkretismus betrachtet wird. ${ }^{8}$

\footnotetext{
5 Vgl. zu diesem Terminus Tabouret-Keller (1969: 306).

6 Zur Opposition „rigide“ vs. ,fluide“ Diglossie vgl. Pauwels (1986: 15).

7 Vgl. zu dieser Terminologie Dorian (2009: 552) und Eller-Wildfeuer (2013: 101f.).

8 Diese terminologische Distinktion kann naturgemäß keine absolute Trennschärfe aufweisen, da die lebensweltliche sprachkommunikative Realität oft fluide und dynamische Sprachphänomene bietet.
} 


\section{DIE EMPIRISCHE MATERIALGRUNDLAGE UND DAS EINGESETZTE VERFAHREN}

Die im Blick stehenden authentischen Diskurssegmente gehen auf direkte (,face-toface“) Kommunikation - nach der Gesprächstypologie von Huneke/Steinig (2013: 95) auf sog. Kontaktgespräche - zurück. Hierzu ist von Bedeutung, dass es sich um gruppeninterne Kommunikation, um Mündlichkeit und gesprochene Sprache handelt mit allen ihren Merkmalen wie Konstruktionsbrüchen, vielen Abtönungen, Pausen, Wiederholungen usw. Die für den Beitrag ausgewerteten Daten entstammen allesamt fränkischbasierten Dialekten aus den folgenden Orten der „Schwäbischen Türkei“: Hetfehell (ung.: Hetvehely), Nadasch (ung.: Mecseknádasd), Tiedisch (ung.: Töttös) und Wieland (ung.: Villány). Der zeitliche Umfang der Audioaufnahmen beträgt insgesamt 6:22:20, die entsprechenden Transkripte ${ }^{9}$ der 15 Gespräche umfassen zusammen 467 Seiten. Das ganze Material ist in unserer Datenbank unter www.uzsk.de abruf- und einsehbar.

Es handelt sich um eine theoriegeleitete, primär empirische Untersuchung: Im Rahmen eines induktiv orientierten korpusbezogenen Herangehens wurde das Datenmaterial in einem Bottom-up-Verfahren beschrieben und expliziert.

\section{BEFUNDLAGE: SPRACHKONTAKTE, UMBRÜCHE UND EROSIONSMOMENTE}

Aufgrund der grundsätzlich dynamischen Verfasstheit donauschwäbischer sog. Sprachinselmundarten konnte die Auseinandersetzung mit den eruierten natürlichen Daten eine Spannbreite unterschiedlicher Sprachphänomene zutage fördern.

\subsection{Minderheitensprache Deutsch als mundartliche Varietät}

Es konnten viele Dialektbesonderheiten erschlossen werden, z. B. das (veraltete) Dialektlexem Sinnerin (Wieland, E_0075, S. 10f.) ${ }^{10}$ :

(1)

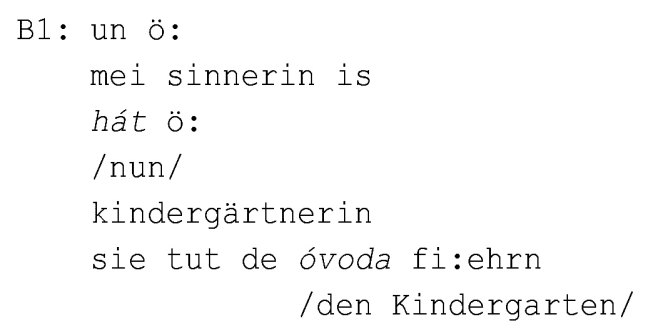

9 Ausführliche Informationen über Anlage und Vorgehensweise des eigens von uns entworfenen Notationssystems, das auf dem Minimaltranskript von GAT 2 basiert, finden sich im Beitrag von Földes (2016b: 176 ff.).

10 Zur Bezeichnung der Belegstellen: Angegeben werden der Untersuchungsort (hier: Wieland), der Name des Datenbankeintrags (hier verkürzt: E_0075, in der Datenbank vollständig: UZSK_E_0075) und die Seitenzahl der Fundstelle in dem in der Datenbank unter www.uzsk.de abrufbaren Transkript (hier: S. 10f.). Alle folgenden Belegstellen werden analog markiert. 
Sinnerin ist eine Formvariante von Söhnerin mit der im Deutschen Wörterbuch von Jacob Grimm und Wilhelm Grimm (1991: Spalte 1423) belegten Bedeutung ,frau des Sohns, schwiegertochter (besonders im südwestdeutschen gebiete, neben söhnin [...]) [...] söhnerin oder sühnerin heist an einigen Orten die schnur". Söhnerin und Schnur werden auch in modernen Wörterbüchern mit dem Vermerk „veraltet“ bzw. „,noch landsch." mit der Bedeutungsangabe ,Schwiegertochter" angegeben (z. B. Dudenredaktion/Scholze-Stubenrecht 1999: 3593 bzw. 3421).

\subsection{Minderheitensprache Deutsch als Kontaktinkubator: Prozesse und Ergebnisse}

Am zahlreichsten und vielgestaltigsten traten im Korpusmaterial Mehrsprachigkeitsphänomene (vor allem Prozesse und Folgen von Sprach- und Kulturkontakten) in Erscheinung.

\subsubsection{Transferenzen}

Das untersuchte Kontaktdeutsch hat sich gleichsam als Hybriditätsinkubator erwiesen, daher soll laut der in Abschnitt 2 genannten Typologie zunächst auf die ermittelten Hybriditätserscheinungen kurz eingegangen werden. Spätestens seit Matras (2009: 146 ff.) ist bekannt, dass zwischensprachliche Transferenzen in verschiedenen Bereichen auftreten können: Transferierbar sind u. a. (a) konkrete Sprachformen, (b) abstrakte Strukturmuster, (c) Bedeutungen und (d) Gebrauchskontexte für Wörter oder Strukturen. Aus Umfangsgründen beschränkt sich dieser Aufsatz schlaglichtartig auf die Betrachtung einiger ausgewählter Phänomentypen. Auf diese Basis aufbauend können demnächst feinkörnigere Studien mehr Details aufdecken und diese ausführlicher erläutern.

Im „Spezialkode“ der Ungarndeutschen begegnen einem regulär hybride Sätze bzw. Sequenzen, wie tut sie korrepetálni, matek und es szakmai usw., vgl. Beleg (2) aus Hetfehell mit einem hessisch-rheinfränkischen Basisdialekt (E_0066, S. 37f.):

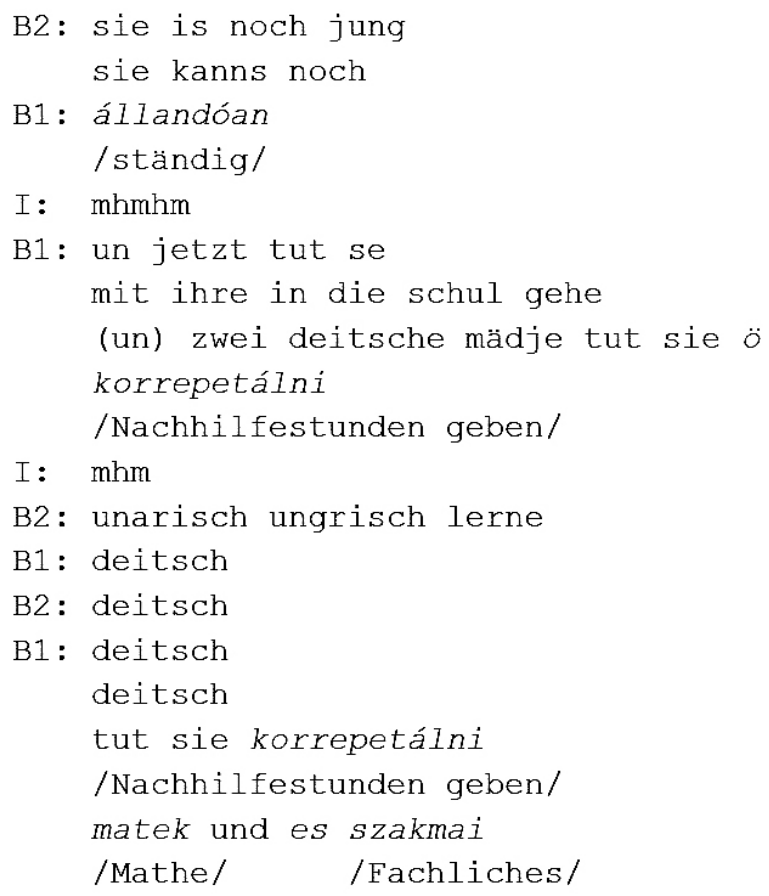


In manchen Sequenzen lässt sich ein gekonntes Balancieren zwischen den beiden Sprach(varietät)en beobachten, Wieland, E_0072, S. 13:

(3) hát sog ich gergő mér nem

/ / warum nicht/
host noch net tovább noch gelernt
/weiter/

noch weider

Die Aussage der 101-jährigen (!) Gewährsperson kann gewissermaßen als „Paradebeispiel“" für das Zusammenspiel der beiden Sprachen gelten: (1) Die syntaktischen Regeln des Deutschen werden hier trotz der ungarischen Transferenzelemente eingehalten, also: „Nun sage ich Gergő [ein männlicher Vorname], warum nicht hast [du] noch nicht weitergelernt? Noch weiter." Die Wortstellung spiegelt also ein deutsches Muster wider, nämlich einen Spannsatz mit einem finiten Verb an letzter Stelle. (2) Auch das hybride Kompositum továbbgelernt (,weitergelernt') ist grammatisch korrekt, es folgt allerdings ungarischen semantischen Regularitäten, denn statt des Verbs továbbtanul (,weiterlernen') würde man im Deutschland-Deutschen in Bezug auf Hochschulen eher studieren sagen. (3) Im Schlussteil wiederholt die Sprecherin das Adverb weider (,weiter') auf Deutsch, was als bilinguale Dopplung betrachtet werden kann, ${ }^{11}$ vgl. Abschnitt 4.2.2.

Es überrascht nicht, dass im Material - neben den hier nicht zu thematisierenden phonologischen - die lexikalisch-semantischen Transferenzen am stärksten vertreten sind. Dabei erfasst der areale Sprachkontakt zuerst Lexeme außerhalb des Kernlexikons, dann den gesamten Wortschatz des Dialekts aufgrund der zunehmenden Dominanz des Ungarischen und der damit einhergehenden Marginalisierung der Minderheitensprache. ${ }^{2}$ Belege sind z. B.:

(4) anyakönyvvezetö (,Standesbeamtin', Hetfehell, E_0067, S. 17);

(5) gyüszüvirág (,Fingerhut', Tiedisch, E_0119, S. 22);

(6) hittan (,Religionslehre', Nadasch, E_0142, S. 54 sowie Tiedisch, E_0068, S. 30);

(7) igazgató (,Direktor', Tiedisch, E_0119, S. 31);

(8) müvésztelep (,Künstlerkolonie', Wieland, E_0071, S. 19 und S. 20);

(9) szociológus (,Soziologe', Tiedisch, E_0119, S. 34) und

(10) tekercselt (,gespult, gewickelt‘, Wieland, E_0083, S. 13).

Belege für den Transfer von Elementen des Grundvokabulars sind u. a.

(11) unoka (,Enkel', Wieland, E_0072, S. 35);

11 Eine andere Interpretation wäre, dass es sich um eine nachträgliche Übersetzung von tovább handelt, weil der Sprecherin bewusst ist, dass die Hauptsprache der Interaktion Deutsch ist, ihr aber nicht gleich das korrekte deutsche Wort eingefallen ist. Deshalb übersetzt sie es dann im Nachhinein, um ihre Orientierung an der aktuellen Interaktionssprache Deutsch kenntlich zu machen.

$12 \mathrm{Zu}$ ähnlichen Beobachtungen gelangt auch z. B. Knipf-Komlósi (2011: 133 ff.) aufgrund ihrer empirischen Studie über die deutsche Minderheitensprache in der Batschka. 
(12) alig (,kaum', Hetfehell, E_0066, S. 4 und S. 5);

(13) föztem (, ich habe gekocht', Nadasch, E_0142, S. 61) und hogyan (,wie', Nadasch, E_0142, S. 33).

Der absolute Spitzenreiter ist der Diskursmarker

(14) hát (etwa: ,dann, nun'), der in sämtlichen Dialogen aus allen Untersuchungsorten gehäuft auftritt; als Beleg könnte z. B. das Transkript Wieland, E_0072 dienen, in der dieses Transferlexem 253-mal vorkommt.

Manche Wörter werden nicht korrekt transferiert, was wahrscheinlich auf Unsicherheiten in der Sprachkompetenz der Sprecher hinweist, z. B.

(15) nyuszikaszáj (wörtlich: „Häschenmaul“, also korrekt und adäquat: nyúlszáj „Hasenscharte', Hetfehell, E_0066, S. 39).

Eine Kontaktwirkung zeigt sich auch darin, dass sehr viele Internationalismen/Europäismen bzw. Wörter fremder Herkunft, die es in beiden Sprachen in ähnlicher Form gibt, nach den Artikulations- bzw. Aussprachekonventionen des Ungarischen vorkommen. Das zeugt davon, dass diese Sprachzeichen über die Struktur der Umgebungssprache Ungarisch in die deutsche Dialektvarietät Eingang finden, z. B.

(16) gáz (,Gas', Tiedisch, E_0119, S. 17f.);

(17) generáció (,Generation', Hetfehell, E_0067, S. 13 und S, 14, Wieland, E_0071, mehrfach S. 20) und

(18) traktor (,Traktor', Nadasch, E_0142, S. 27 und S. 29, Wieland, E_0071, S. 5, S. 17 und mehrfach S. 19).

Einen großen Teil von ihnen machen die Kulturwörter und die "Sachmodernismen“ aus, die in der Forschungsliteratur in Bezug auf andere Dialektlandschaften bereits mehrfach thematisiert wurden, z. B. von Knipf-Komlósi (2011: 135 und 138).

Es werden nicht nur Simplizia transferiert, sondern auch, um nur drei Beispiele zu nennen:

(a) komplexe Benennungen als Wortgruppen, ${ }^{13}$ vgl. Wieland, E_0072, S. 9:

(19) momentan muss ich in orr-fül-gégészet

/Hals-, Nasen- und Ohrenheilkunde/

(b) Adjektiv-Substantiv-Verbindungen, vgl. Wieland, E_0072, S. 10:

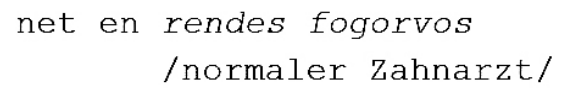

13 Die von der Sprecherin gewählte ungarische Wortverbindung orr-fül-gégészet (wörtlich: ,NasenOhren-Halsheilkunde') ist nicht stimmig, eigentlich müsste es fül-orr-gégészet heißen (also wörtlich: ,Ohren-Nasen-Halsheilkunde'). 
(c) oder eben mehrteilige Konjunktionen, vgl. Wieland, E_0072, S. 58: ${ }^{14}$

(21) B1: noch be:t ich

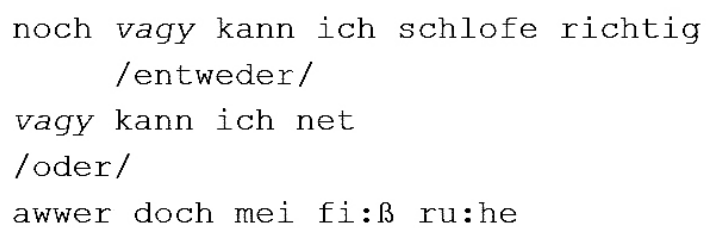

Hier wird die zweiteilige Konjunktion entweder - oder durch ungarisch vagy - vagy (wörtlich: „oder - oder“) ausgedrückt.

Frequent sind auch hybride Substantivkomposita wie

(22) Herzorvos (,Herzarzt, Kardiologe‘ als Determinativkompositum aus deutsch Herz + orvos $=$, Arzt $^{6}$, Wieland, E_0072, S. 9) und von der Wortbildungsart her ebenfalls als hybride Zusammensetzungen einzustufenden Adverbien wie

(23) dehogynet (nach dem Modell dehogynem: ungarisch dehogy + deutsch net ,nicht ${ }^{*}$ im Sinne von ,doch', Wieland, E_0072, S. 6).

Der kommunikative Umgang mit Jahreszahlen weist ebenfalls auf eine ungarische Vorlage hin: ${ }^{15}$ Beispielsweise wird in einem Beleg 1917 nicht mit neunzehnhundertsiebzehn verbalisiert, sondern es folgt ungarischen Mustern, wie folgender Beleg aus Nadasch, E_0070, S. 6 demonstriert:

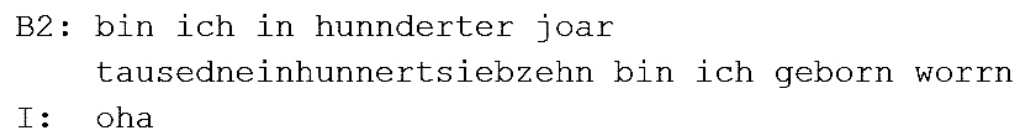

Die fortgeschrittene Symbiose der beiden Sprach(varietät)en wird u. a. auch daran deutlich, dass z. B. bei ungarischen Verben in hybriden oder in sonst einsprachig deutschdialektalen Sätzen die Suffigierung oft durch deutsche Flexive erfolgt. Beim Partizip II wird dem ungarischen Wortstamm das deutsche Partizipialpräfix ge- vorgeschaltet:

(25) gedíszit (,geschmückt‘, Nadasch, E_0142, S. 2),

(26) gekövetelt (,verlangt', Nadasch, E_0142, S. 54 und S. 57),

(27) getelepit und rausgetelepit (,gesiedelt" und , ausgesiedelt', Wieland, E_0075, S. 6 und S. 12) usw.

Noch interessanter sind ungarische präfigierte Verben, die mit deutschen Partikelverben kombiniert werden. Hier ist das deutsche ge-Präfix zwischen dem ungarischen (trennbaren) Verbalpräfix und dem ungarischen Stamm positioniert. Die Endung - $t$ im

14 Dasselbe Phänomen ist auch z. B. in Wieland, E_0072, S. 48 sowie in Hetfehell, E_0066, S. 8 und 21 belegt.

15 Im Ungarischen werden auch die Jahreszahlen von 1100 bis 1999 nach Tausendern (und nicht nach Hundertern) zusammengefasst. 
Auslaut kann sowohl als Teil des ungarischen Wortstammes wie auch als deutsches Dentalsuffix interpretiert werden, vgl. Beleg (28) mit dem Partizip Perfekt fölgedíszít (, aufgeschmückt'), Nadasch, E_0142, S. 1:

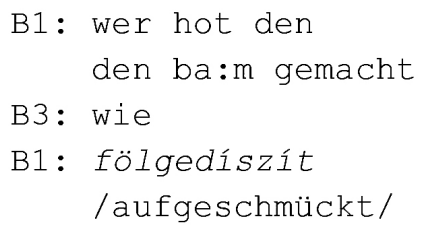

Sehr produktiv ist das Konstruktionsmuster, in dem ungarische Nomina in eine deutsche Präpositionalphrase inkorporiert werden, vgl. Beleg (29) aus Wieland, E_0075, S. 2.

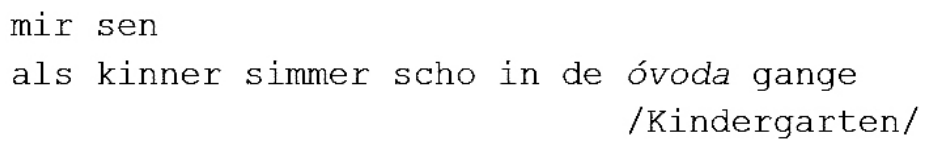

Also in die Óvoda gegangen, d. h. in den Kindergarten gegangen. Ähnliche Beispiele sind aus dem Korpus u. a.

(30) die warn auf gyakorlat, d. h. sie waren im Praktikum, Nadasch, E_0070, mehrfach S. 20 und S. 22;

(31) dass se se uffgenomme hon uff de egyetem, d. h. dass sie sie aufgenommen haben auf/in die Universität, Hetfehell, E_0066, S. 25 und S. 26;

(32) in de bölcsöde gange, d. h. in die Kinderkrippe gegangen, Wieland, E_0075, S. 17.

Von hohem Aufschluss sind die Belege, in denen ein und dieselbe grammatische Funktion zweimal und sogar mit kategorial unterschiedlichen Mitteln ausgedrückt wird. Eine derartige morphosyntaktische Markierung wäre beim Kontakt von zwei modernen indogermanischen Sprachen nicht möglich, da aber Deutsch und Ungarisch typologisch disparate (und genetisch nicht-verwandte) Sprachen sind, konnte z. B. in der folgenden Präpositionalphrase die Ortsbestimmung zweifach bezeichnet werden: Einmal mit der deutschen lokalen Präposition in und ein andermal mit dem ungarischen Superessiv-Suffix ${ }^{16}$-on, also in Martonvásáron (,in Martonvásár'), vgl. Tiedisch, E_0119, S. 42:17

(33) B1: des sin laute obschtgärte un un weigoarde un

B2:

in martonvásáron

16 Grundbedeutung: Position auf einem Bezugsobjekt.

17 Ähnliche Beispiele aus einem anderen ungarndeutschen Ort mit der Basismundart Schwäbisch finden sich mit ausführlichen Interpretationen in Földes (2005: 153 ff.). 
Uneinheitlich ist der Umgang mit den Lexemen ungarischer Provenienz im Hinblick auf ihre grammatische Integration: Mal werden sie den Regeln der deutschen Sprachvarietät gemäß flektiert (als Regelfall), mal weisen sie ungarische Kasussuffixe auf (z. B. Beleg Nr. 34 aus Wieland, E_0072, S. 26) oder bleiben in ihrer ungarischen Nennform und erhalten weder eine ungarische noch eine deutsche Endung, vgl. Beleg (35) aus Hetfehell, 0066, S. 31.

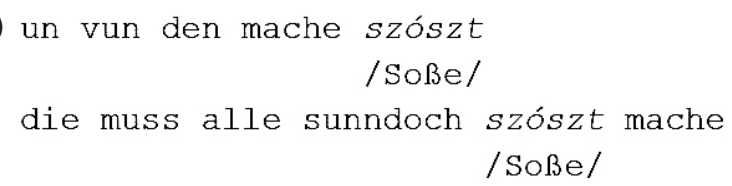

$$
\begin{aligned}
\text { wie sie die ungrisch jogosítvány hot krie:t } \\
\text { /Führerschein/ }
\end{aligned}
$$

Im Beleg (34) wird die ungarische Akkusativendung $-t$ bei den beiden Vorkommen von szósz (,Soße') verwendet, während im Beleg (35) das Lexem jogosítvány (,Führerschein') ohne Flexionsendung steht.

Noch mehr fällt das syntaktisch-lexikalische Phänomen der hybriden tun-Periphrase unter Rekurs auf ungarische infinite Verbformen auf, z. B. aus Wieland, E_0072, S. 14:

$$
\begin{aligned}
\text { wos alle tog traktorozni tut } \\
\text { /Traktor fahren/ }
\end{aligned}
$$

Weitere Belege sind u. a.

(37) tut végezni, d. h. tut abschließen, Wieland, E_0072, S. 40;

(38) tu eich megkinálni, d. h. tu euch (etwas) anbieten, Nadasch, E_0142, S. 63;

(39) tun tornázni, d. h. tun turnen, Wieland, E_0072, S. 57 und S. 58. Vgl. auch die Belege (1) und (2).

Auch in semantischer Hinsicht (Bedeutungsumfang, Unter- bzw. Überspezifizierung etc.) entfaltet Ungarisch seine Strahlkraft, vgl. Beleg (40) aus Hetfehell, E_0067, S. 58 im Sinne von, ich kann es nicht genau sagen ": 18

(40) há des kann ich net pinktlich sage /nun/

Der Beleg (41) zeigt den Gebrauch des Wortes Programm in seiner häufigen ungarischen Bedeutung: ,zu tun haben“ bzw. ,etw. vorhaben', Wieland E_0071, S. 9f.:

(41) B1: is immer programm

B2: immer immer immer immer

18 Dem ungarischen Adverb pontosan kann im Sprachsystem des Deutschen sowohl pünktlich als auch genau entsprechen. 
Man kann beobachten, dass das Gros der salienten Belege mit ungarischen Bauplänen, Konstruktions- bzw. Formulierungsmustern zu erklären ist; folglich treten in Bezug auf Rektionen, Kollokationen, syntaktische Strukturen etc. vielgestaltige implizite Kontaktmanifestationen auf, man könnte sie auch zwischensprachliche Parallelstrukturiertheiten nennen z. B.

(42) der hot angst von wasser, Tiedisch, E_0068, S. 17, Standarddeutsch: der hat Angst vor dem Wasser, aber ungarisch: fél a vizztöl (d. h. wörtlich: ,Angst vom Wasser haben').

(43) die ist auch in krebs gestorwe, Tiedisch, E_0119, S. 28, Standarddeutsch: die ist auch an Krebs gestorben, aber ungarisch: ö is rákban halt meg (d. h. wörtlich: ,auch sie ist in Krebs gestorben').

Da Wortschatzelemente zugleich soziale und kulturelle Phänomene sind, hat man es in vielen Fällen speziell mit kulturell motivierten Kontaktphänomenen zu tun. Beispielsweise Maß- und Mengenangaben wie Dezi(liter $)^{19}$ als ein Zehntel Liter, in Ungarn (und in Österreich) überaus geläufig (Wieland, E_0072, S. 55f.):

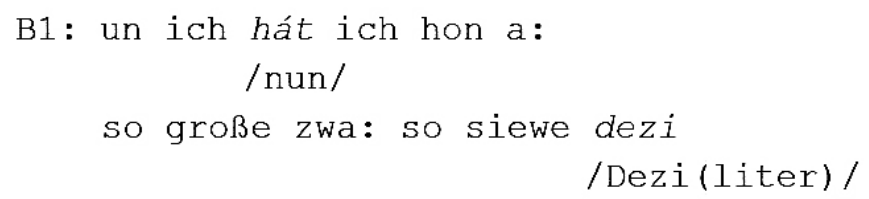

Auch der Namenstag ${ }^{20}$ weist bei den Ungarndeutschen eine kulturbezogene Bedeutung auf (Wieland, E_0075, S. 23):

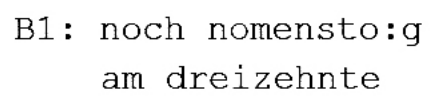

Man kann nicht immer eindeutig entscheiden, ob ein interlinguales Kontaktphänomen vorliegt oder ob die Gründe für die (synchrone) Auffälligkeit innerhalb des Deutschen zu suchen sind. Beispielsweise lässt sich das Kompositum

(46) Fliegerplatz

im Sinne von ,Flugplatz‘ (Wieland, E_0075, S. 10) als Transferenzübersetzung aus dem ungarischen Determinativkompositum repülötér (wörtlich: ,Flieger ${ }^{\star}+$,Platz') deuten.

In anderen Fällen liegt die Annahme nahe, dass Sprachkontaktprozesse am Werk sind, wie z. B. beim Verb

(47) aufsteigen

in der Bedeutung , aufstehen': z. B. Hetfehell, E_0066, S. 40 und Wieland, E_0083, S. 4, da das entsprechende ungarische Lexem felkel von seiner Morphemstruktur her mit dem deutschen Verb aufsteigen korrespondiert. Das Grimmsche Wörterbuch belegt

19 Als Lemma auch z. B. im Wörterbuch der donauschwäbischen Lebensformen verzeichnet, vgl. Gehl (2005: 184).

20 Auch bei Gehl (2005: 711) gebucht. 
allerdings: ,aus dem bette, vom tische aufsteigen, oder beidemal blosz aufsteigen für aufstehen" [Hervorhebungen im Original] (Grimm 1984: Spalte 748). Insofern müssen diese Belege nicht unbedingt als Kontaktergebnisse interpretiert werden, womöglich handelt es sich um ältere Formen bzw. Bedeutungen.

Für intrasituative Variation liegen auch Beispiele vor: Gelegentlich schwanken in den Diskursen die (ungarn)deutschen und die ungarischen Lexeme selbst für identische Referenzobjekte. Beim folgenden Beleg könnte man noch denken, dass der Sprecherin aus Gründen der Geläufigkeit zunächst das ungarische Wort otthon einfällt und sie erst einige Minuten später auf das deutsche Äquivalent Altersheim kommt, ${ }^{21}$ Nadasch, E_0070, S. 3 und S. 6:

(48) noch bin ich do herkumme

in den otthon

/ins Heim (Altersheim)/

bzw.

(49) in budapest

un noch so bin ich heimkumme in

altersheim

Auffälliger ist, dass in einem anderen Dialog eine Sprecherin zuerst das korrekte deutsche Lexem verwendet, dann aber das ungarische Pendant tévé (mit ungarischer Aussprache), vgl. Wieland, E_0072, beide S. 57:

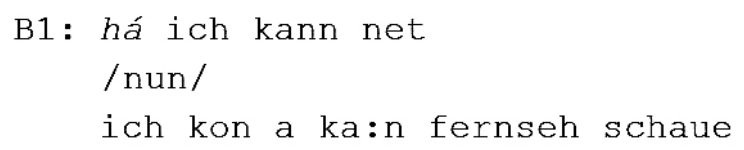

bzw.

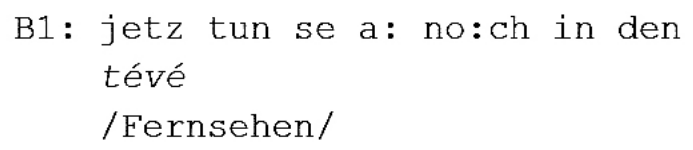

Diese und andere Schwankungen können einerseits als lexikalische Parallelformen (nativ und transferiert) aufgefasst werden, andererseits demonstrieren sie, dass die mehrsprachige Kompetenz und Performanz ausdrückliche Prozessualität und Dynamik aufweisen, nicht geradlinig sind und mitunter auch Brüche und Widersprüche oder zumindest Inkonsequenzen zeigen. ${ }^{22}$

Der Sprachkontakt kann mitunter zu Frequenzsteigerungen führen: Das Adverb (52) vielmal

21 Eine andere Sache ist, dass sie dann etwas später wieder otthon sagt.

22 Auch in der phonetischen Realisierung gibt es Schwankungen: Dieselbe Sprecherin verwendet innerhalb einer Dialogsequenz zuerst schwäwisch, dann schwäbisch und schließlich schwawisch (Hetfehell, E_0067, S. 5). 
mag auf den ersten Blick als veraltet gelten, wie es z. B. im Duden (Dudenredaktion/Scholze-Studenbrecht 1999: 4324) gekennzeichnet ist. Eine Berücksichtigung der Kontaktsprache Ungarisch, in der dieselbe Struktur gängig ist: sokszor, legt jedoch die Annahme nahe, dass hier der Sprachkontakt den Ausschlag gibt, zumal in den hier analysierten 15 Gesprächen diese Form vielmal nicht weniger als 31-mal vorkommt.

Gelegentlich gibt es im Datenkorpus zudem Belege, denen zwar ein deutsches Konzept zugrunde liegt, das aber mit ungarischen Redemitteln realisiert wird, was für ein hohes Maß an kultureller, identitätsbezogener und sprachlicher Überkreuzung bzw. Überblendung spricht; vgl. Beleg (53) aus Wieland E_0072, S. 59f.:

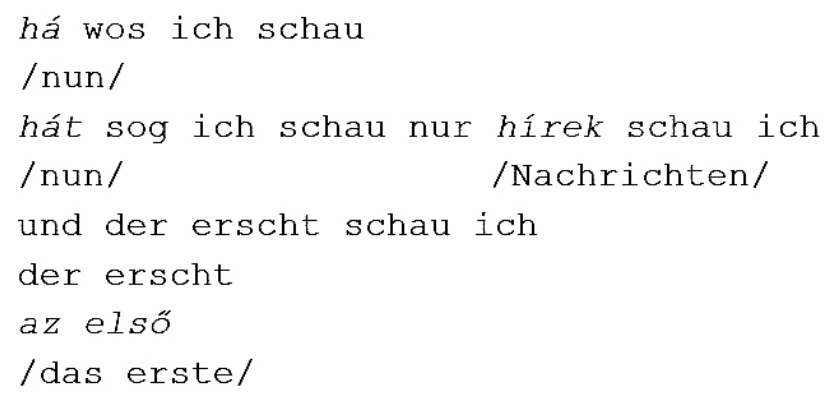

Im Ungarischen würde man wohl kaum első (,das Erste') sagen, vielmehr wäre egyes (, der Einser') geläufig, sodass hinter dieser ungarischen Sprachmanifestation ein deutsches Modellkonzept zu vermuten ist. In ähnlicher Weise dürfte die hybride Kombination vagy $S^{23}$ eigentlich dem deutschen Nominationsmuster oder so folgen (Hetfehell, E_0066, S. 13):

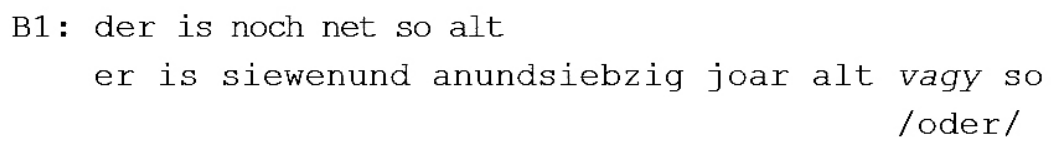

B1: der is noch net so alt er is siewenund anundsiebzig joar alt vagy so /oder/

Eine gesonderte Erwähnung verdienen die ungarndeutschen hybriden Irrelevanzkonstruktionen mit der ungarischen Vorsilbe akár-. Sie zeichnen sich durch hochgradige Produktivität aus und sind gleichsam im gesamten donauschwäbischen Siedlungsraum verbreitet (vgl. Földes 2005: 168f. und Szabó 2010: 381). Vgl. z. B. Belege (55) und (56) aus Nadasch, E_0070, S. $25^{24}$ und E_0142, S. 42:

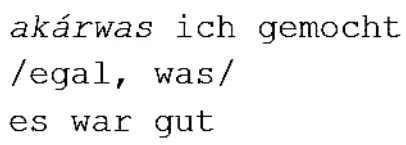

23 Die nebenordnende Konjunktion vagy = wörtlich: ,oder‘.

24 Analog auch in Belegen aus Tiedisch, E_0119, S. 12 und Hetfehell, E_0067, S. 12. 


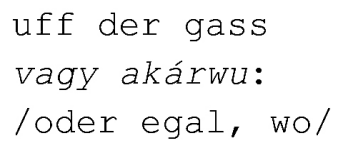

Die durch Zusammensetzung konstruierten ungarischen Indefinitpronomina werden vom bilingualen Sprachträger re-etymologisiert; dadurch tritt ihre Motivation klar in Erscheinung: Das Präfix akár ${ }^{25}$ wird aus der Zusammensetzung isoliert und mit den deutschen Fragepronomina was bzw. wo verknüpft. Es gibt auch eine ganze Wortbildungsreihe: akárwer, akárwie, akárwann etc. In den Diskursen werden sie deutsch flektiert, nicht nur in pronominaler, sondern auch in konjunktionaler Funktion. Sie können als Irrelevanzkonditionalien aufgefasst werden, bei denen ein $w$-Wort mithilfe der Partikeln auch und/oder immer generalisiert wird, also entspricht akárwas in etwa der Wortgruppe was auch immer. Diese Irrelevanzkonditionalien nehmen eine Zwischenstellung zwischen Konditionalität und Konzessivität ein (vgl. Szabó 2010: 385).

Vermutlich erfreuen sich die mit dem Präfix akár- gebildeten Komposita großer Verbreitung, weil die als Muster dienende ungarische Konstruktion sprachlich viel einfacher, ökonomischer und transparenter ist als die entsprechenden analytischen Ausdrucksweisen des Deutschen ( $w$-Fragewort + auch immer + oft noch ein Nebensatz). Der Transparenz fällt eine besondere Bedeutung zu, weil sie dem kognitiven Prozess entgegenkommt und damit den zwischensprachlichen Transfer begünstigt (Földes 2005: 170f.). Die kommunikative Verwendung dieser hybriden Irrelevanzkonditionalien in großem Umfang ist ein Indiz dafür, dass diese Strukturen im mentalen Lexikon der Sprecher bereits als neutrale Grenzgänger fungieren und in ihren Kontroll- sowie Monitorinstanzen in deutschsprachigen Zusammenhängen nicht mehr als „fremd“ empfunden werden. Hybriditäten dieser Art setzen eine besondere sprachliche Kompetenz voraus, eine „Metagrammatik, die sprachliches Wissen umfasst, über das Monolinguale (oder Bilinguale, die ausschließlich in einem monolingualen Modus, einmal in Sprache A und einmal in Sprache B operieren) nicht verfügen“ (Auer 2009: 93).

\subsubsection{Kode-Umschaltung und bilinguale Dopplung}

Kommunikativer Synkretismus (siehe Abschnitt 2) offenbart sich in den erschlossenen Sprechproben grundsätzlich in Form von Kode-Umschaltung und bilingualer Dopplung.

Kode-Umschaltung hat als Äußerungsformat bzw. als bilingualer Gesprächsstil viele Gesichter. Auf eine ausführliche Auseinandersetzung mit der konzepttheoretischen Basis dieses komplexen Phänomens sei an dieser Stelle verzichtet; zentrale Erkenntnisse bietet z. B. die Monographie von Gardner-Chloros (2009). Im vorliegenden Aufsatz wird „Code-switching“ im Anschluss an Müller (2017: 9) als „,sanfter, ohne Häsitationen und Pausen vonstattengehender Wechsel zwischen zwei oder mehreren Sprachen“ begriffen. ${ }^{26}$ Viele Kode-Umschaltungssequenzen zeigen ein harmonisches Bild; dabei verlangen besonders die intrasentenziellen Wechsel vom Sprecher intakte

25 Sinngemäß: egal.

26 Dabei ist diese Begriffsbestimmung insofern zu spezifizieren, als damit nicht nur ein Wechsel zwischen Einzelsprachen, sondern auch zwischen Varietäten einer Sprache gemeint werden kann. 
sprachkommunikative Fertigkeiten in beiden Sprachen: In diesen Fällen werden an den Schaltstellen die syntaktischen Regeln beider Sprachen weitgehend beachtet, es entstehen kaum Konflikte zwischen den aufeinandertreffenden grammatischen Strukturregularitäten. Vgl. Beleg (57) Hetfehell, E_0066, S. 45:

B2: ich ma:n der józsi bácsi kommt net

B1: hát fekszik há beteg

/nun, er ist bettlägerig, er ist nun krank/

tegnap is má feküdt

/gestern hat er auch schon im Bett gelegen/

B2: hat sich schun

ja

I: $\mathrm{mhm}$

B2: hat sich schon hi:gelegt

Aus vielen Belegen lässt sich hingegen eher auf Kompetenzschwierigkeiten schließen. Diese Umschaltungsitems, bei denen also unzureichendes Performanzvermögen den Wechsel auslöst, kann man als code-shifting (Silva-Corvalán 1983: 85) oder als Kodemischung (Auer 1999: 309) explizieren, vgl. Beleg (58) aus Tiedisch E_0068, 4f.:

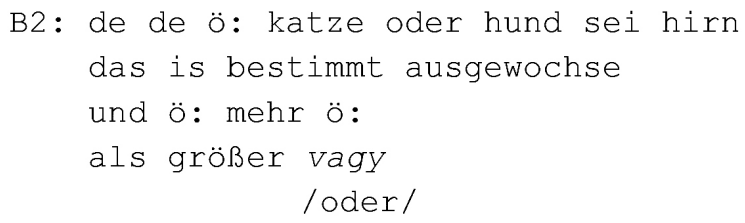

I : $\quad m h m$

B2: hogy mondjam

/wie soll ich sagen/

öh :

üwwerhaupt der hund

der hot so e großes hirn

als der mensch

I: $\quad m h m$

B2: un ö: er is ö: sehr ö: wo

I: ausgeprägt

B2: ausgprägt un ö:

un un

nem tudom megmondani neked

/ich kann es dir nicht sagen/

Szilvi most hogy mit akartam

ISilvie was ich denn wolltel

I: na die sind klug ne 
Man erkennt hier, dass die Sprecherin B2 ständig nach Wörtern sucht und kaum eine Balance zwischen den zwei Sprachen findet.

Neben offensichtlichen Sprechproduktionsschwierigkeiten tritt im Kode-Umschaltungsbeleg (59) aus Hetfehell, E_0067, S. 52 eine zwischensprachliche Kompromissform als hybrides Produkt auf, nämlich das Adverb svájcerosan. Bei dieser interlingualen Verknüpfung formnaher Elemente findet eine Koaktivierung bei der Sprachproduktion statt: Das deutsche Lexem schweizerisch wird dem phonologischen System des Ungarischen angepasst und mit einem Suffix des semantischen Kasus Essiv-Modal27 ergänzt. Die sprachübliche ungarische Form wäre svájciasan gewesen.

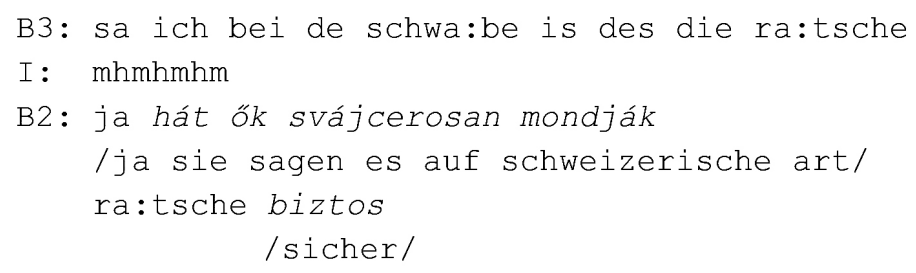

Für bilinguale Dopplungen als besondere Art von Synkretismus lieferte die Datensammlung ebenfalls zahlreiche Belege. Es handelt sich hier um eine Äußerungsstrategie, bei der die vollständige Mitteilung oder ein Teil von ihr im Anschluss in der anderen Sprache nochmals ausgedrückt wird, also eine Wiederholung desselben Inhalts in zwei Sprachen erfolgt (vgl. Földes 2005: 239 ff.):

Oft betrifft die Dopplung nur einzelne Konstituenten, z. B. im Beleg aus Wieland, E_0072, S. 42:

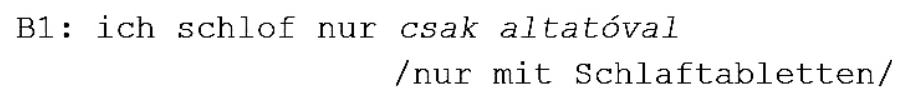

Sie kann sich aber auch auf ganze Wortgruppen beziehen, vgl. Nadasch, E_0070, S. 2:

(61) die war derha:m

die hot noch misse mei großmottr

mach

oha

nehéz idók voltak

/das waren schwere zeiten/

des warn schlimme zeide gell

27 In der Funktion einer Modalbestimmung bei Adjektiven, ausgedrückt mit den Suffixvarianten $-n /-a n /-e n$. 


\subsubsection{Sonstige kommunikative Praktiken}

Außerdem fallen als Folge von Mehrsprachigkeit nicht lediglich die unter 4.2.1 und 4.2.2 illustrierten Variationsmuster auf, sondern auch z. T. recht subtile Vermeidungsstrategien, Übergeneralisierungen $u$. a. Es heißt: Aus kontakt- bzw. variationslinguistischer Sicht ist nicht nur das relevant, was der mehrsprachige Diskursakteur sagt und wie er das sprachlich umsetzt, sondern auch, was und warum er etwas nicht sagt, warum er sich bestimmter Zeichen(kombinationen) der einen Sprache gar nicht oder kaum bedient. Eine Erfassung dieser Phänomene wäre allerdings nur durch aufwändige Frequenzuntersuchungen etc. möglich (Földes 2013: 133).

\section{EROSIONS- BZW. ATTRITIONSPROZESSE}

\subsection{Deutsch als Minderheitensprache zwischen Kontaktkreativität und Sprach- verschleiß}

Ein Teil der obigen Belege kann im Rahmen der lebendigen Dynamik multilingualer Sprachkompetenz als kreativer Umgang mit beiden Sprach(varietät)en interpretiert werden (vgl. die Belege 3, 55 und 56). Dabei ist aber nicht zu übersehen, dass manche Diskursbeispiele (z. B. die Belege 58 und 59) eher Unsicherheit und/oder Schwund in den sprachkommunikativen Fertigkeiten in Bezug auf das Deutsche (und gelegentlich auch in Bezug auf das Ungarische) nahelegen. Es lässt sich jedoch nicht einfach entscheiden, was kontaktbedingter Sprachwandel, was individuumspezifische Spracherosion (language attrition) und was intergenerationeller Sprachverlust (language shift) ist, vgl. de Bot/Weltens (1991).

\subsection{Spracherosion: Konzeptualisierungen, Manifestationen und Interpretationen}

Für das teilweise oder das totale Vergessen einer Sprache sollen hier im Anschluss an Schmid (2011: 3) die Termini Spracherosion oder Attrition (engl. language attrition) verwendet werden. ${ }^{28}$ Die Strukturen der einst erworbenen, aber immer weniger gebrauchten Sprache gehen mit der Zeit zurück und der Sprecher kann sich dieser somit nicht mehr bedienen: Das Individuum büßt seine Sprachkompetenz ein (vgl. Wildfeuer 2017: 199). Es gibt hierfür in der Forschungsliteratur unterschiedliche Konzeptualisierungen: Nach Riehl (2014: 93) und Köpke (2019: 350), deren Modell auch der vorliegende Aufsatz folgt, ist zwischen (1) dem individuellen (= Spracherosion, Attrition) und (2) dem gesellschaftlichen (= Sprachwandel, Sprachwechsel) „Vergessen“ einer Sprache zu differenzieren. Bei Wildfeuer (2017: 199f.) hingegen gibt es zum einen (1) den „Sprachverlust“, welcher das Aufgeben einer Varietät bedeutet und zum anderen (2) den „Sprachtod“, welcher sich auf das globale Verschwinden eines ganzen Sprachsystems bezieht. ${ }^{29}$ Beide Phänomene werden von ihm jeweils in „Sprachverschiebung“ (gesellschaftlich) und „Sprachverfall“ (individuell) untergliedert. Die Sprachverschiebung innerhalb des „Sprachverlustes“ kann

28 Über die Problem- bzw. Forschungsgeschichte referiert z. B. Anstatt (2011: 7 ff.)

29 Viele Forscher nehmen keine Differenzierung vor und sprechen im Hinblick auf die eingeschränkte Vitalität des Deutschen als Minderheitensprache einfach von „Sprachverlust“, z. B. Foschi Albert (2018: 113). 
etwa mit Riehls ,Sprachwandel` gleichgesetzt werden. Der Sprachverfall innerhalb des „Sprachverlustes“ kommt Riehls ,Spracherosion“ bzw. ,Attrition“ nahe. ${ }^{30}$

Sprachstrukturelle und funktionale Spracherosion ist - wie de Bot/Lowie/Verspoor (2007: 7) ausführen - ein hochkomplexer und zum Teil unvorhersehbarer Restrukturierungsprozess, welcher graduell verläuft (Köpke 2019: 365). ${ }^{31}$ Er beruht auf Mehrsprachigkeitseffekten, die in Abschnitt 4.2 diskutiert wurden. Es gibt zwei Phänomene der Attrition: (1) Erscheinungen, welche durch einen Mangel an Gebrauch verursacht, und (2) Entwicklungen, die durch Sprachkontakt induziert werden (vgl. Riehl 2014: 95). Es ist anzunehmen, dass im analysierten Sprach-Mikrokosmos beide Typen miteinander zusammenhängen. ${ }^{32}$ Anzeichen für eine beginnende Attrition sind lexikalisch-semantische Fehloptionen, Ausdrucksfehler, Wortsuche und nicht-funktionale Kode-Umschaltungen, vgl. z. B. Belege (40) und (58).

Unter Sprachwechsel oder auch Sprachwandel (engl. language shift) wird hier die Sprachumstellung einer Gemeinschaft verstanden (vgl. Schmid 2011: 3). Dieser Prozess hängt eng mit Attrition zusammen, denn diese beginnt bei einzelnen Sprechern und verbreitet sich in der Sprachgemeinschaft (Riehl 2016: 254). Aufgrund der Kombination von Attrition in einer Generation und dem Wechsel der Generationen können so Sprach(varietät)en verloren gehen: Die folgenden Generationen bekommen eine von Sprachvereinfachungsprozessen geprägte Varietät als Input, welche zur Kommunikation nicht in vollem Maße ausreicht. Dieses Problem findet sich oftmals in Migrationssituationen, wenn die Sprecher die Erstsprache nicht in ihren literaten Formaten erwerben können und/oder es kaum Möglichkeiten gibt, die Sprache lebensweltlich anzuwenden (Riehl 2014: 93 und 2016: 254). ${ }^{33}$

Für Erosionsprozesse können verschiedene Erklärungen postuliert werden. Zugriffsprobleme auf sprachliche Items (etwa Wortfindungsschwierigkeiten), für die im Obigen manche Belege stehen, vgl. (58), lässt sich z. B. die Schwellenwerthypothese (engl. activation threshold) von Paradis (2004) heranziehen. Diese besagt, dass ein

30 Bei der Attrition gibt es nach Hamers und Blanc (2005: 77-79) drei verschiedene Arten: (a) die umweltbedingte, (b) die altersbedingte und (c) die pathologische. Die umweltbedingte Attrition tritt durch einen eingeschränkten/geringen Gebrauch der Sprache auf (das ist also der Typ, der im vorliegenden Fall von Relevanz ist), während die altersbedingte aufgrund von Alterungsprozessen und die pathologische aufgrund von Krankheiten oder Traumata zum Vorschein kommt. Die umweltbedingte Attrition betrifft vorrangig mehrsprachige Menschen in einem anderssprachigen Umfeld. Die Sprache (meist die Erstsprache) wird zu wenig vom Sprecher benutzt, da die dominante Sprache der Umgebung Medium der täglichen Kommunikation ist (Schmid 2011: 4). Somit kommt es zur Schwächung der Position und zum Abbau des Systems der Erstsprache.

31 Der variable Input und der schwindende Gebrauch leisten also Simplifizierungs- und Restrukturierungsprozessen Vorschub.

32 Mithin spielen Prozesse der Spracherosion (aus Sicht des Individuums) und des Sprachabbaus (aus Sicht des Kollektivs) ineinander; der Sprachabbau vollzieht sich allmählich über mehrere Sprechergenerationen.

33 Im Gegensatz dazu bezeichnet Wildfeuer (2017: 199) den Sprachwechsel als „Sprachverschiebung“. Dieser Prozess findet nach Wildfeuer während eines Generationswechsels in Richtung der dominierenden Kontaktsprache statt. 
Item durch eine bestimmte Anzahl positiver Impulse seine neurale Grundlage erlangen kann. Das Hören und Benutzen eines Wortes stellt einen Impuls für den Kommunikator dar und die Anzahl der Impulse, die für die Verfügbarkeit des Items notwendig sind, ergibt den Schwellenwert. Somit kommt der Inputfrequenz große Bedeutung zu: Der Input hinterlässt Gedächtnisspuren und wirkt sich auf das neuronale System und die internen Prozesse aus; das interne System verantwortet die Nutzbarmachung des Inputs im kommunikativen Haushalt (vgl. de Bot 2018: 144). Sobald ein Item im Kopf des Kommunikators durch einen Impuls aktiviert wird, sinkt sein Schwellenwert und es werden weniger Impulse benötigt, um es erneut zu aktivieren. Nach jeder Aktivierung wird demzufolge der Schwellenwert herabgesetzt, er steigt danach allerdings ein wenig an, sobald die Impulse wieder abnehmen (Paradis 2004: 28). Einheiten, mit denen der Sprecher bereits hinreichend Erfahrung gemacht hat, können ohne hohe kognitive Anstrengung aktiviert werden; solche Einheiten sind im Gedächtnis des Sprechers schon so verankert (engl. entrenched), dass sich ihre Aktivierung bereits zu einer automatisierten Routine entwickelt hat (Schmid 2007: 118). ${ }^{34}$ Im Sinne des Frequenz-Prinzips korreliert die Vorkommenshäufigkeit einer sprachlichen Einheit innerhalb eines Diskurskollektivs mit dem Grad an kognitiver Verfestigung der Einheit im Sprachwissen der Mitglieder desselben Diskurskollektivs (Ziem/Lasch 2013: 103). Im Sinne der Regression von Entrenchment (vgl. Riehl 2015 und 2016) kann man darin mehrere Tendenzen feststellen. Im Spracherwerb werden, wie Riehl darlegt (2015: 286 und 2016: 260), konkrete Konstruktionen zuerst erworben (z. B. Mama gibt der Tochter den Ted$d y$ ) und allmählich abstrakte Schemata entwickelt ( $X$ gibt $\left.Y_{d a t} Z_{a k k}\right)$. Außerdem bleiben konkrete Konstruktionen mit hoher Gebrauchsfrequenz als Einheit/Ganzheit erhalten (die sog. frozen units), vgl. Riehl (2015: 286 und 2016: 260), z. B. in Rente gehen in Beleg (64) aus Tiedisch, E_0119, S. 30:

ower der will net in rente gehn

Es ist aufgrund der Literatur (Riehl 2015: 286; 2016: 260) und der empirischen Befunde des Projekts anzunehmen, dass im Verlauf der Spracherosion abstrakte Schemata zuerst verlorengehen (z. B. die vom Verb zugewiesene Dativmarkierung, vgl. Beleg aus Tiedisch, E_0119, S. 24 mit dem Verb ausweichen):

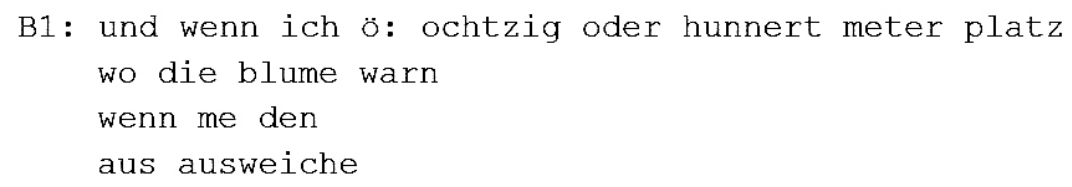

34 Die Anzahl der für die Verfügbarkeit notwendigen Impulse kann nach Paradis (2004: 29 und 31) je nach der Neuheit oder Häufigkeit des Items schwanken. Um den Schwellenwert gering zu halten und um Wortfindungsschwierigkeiten vorzubeugen, muss die Sprache regelmäßig benutzt werden. Wird ein Item nicht aktiviert, so kann der Sprecher nicht mehr darauf zurückgreifen. Attrition ist die Folge einer längeren Aktivierungspause. Durch intensive Nutzung oder durch erneuten Input wird der Schwellenwert geringer und die Attrition kann aufgehalten und die Sprache für den Sprecher wieder zugänglich gemacht werden (Paradis 2004: 28). 
Zudem scheint zu gelten: Konkretere Konstruktionen werden länger im Gedächtnis behalten (z. B. von Präpositionen zugewiesene Dativmarkierung, Riehl 2015: 286, vgl. die Belege 34 und 56) und Einheiten mit einem hohen Grad bestimmter Salienztypen ${ }^{35}$ fordern ebenfalls eine geringere kognitive Anstrengung für den Aktivierungsprozess (vgl. auch Schmid 2007: 119). Im Sinne der dynamischen Systemtheorie erzeugen Sprachkontakte nicht nur bestimmte Effekte, sondern weisen eine völlige Vernetzung und Interaktion miteinander auf (vgl. de Bot 2018: 143 ff.). Folglich bedeutet Erosion „nicht nur den Verlust einzelner Elemente oder Strukturen: Wenn Elemente verloren gehen, kann sich das Gesamtsystem neu organisieren, um zu einem neuen AttraktorZustand zu finden“" (de Bot 2018: 144).

Ob bzw. inwieweit die Erosions-, Kulturtransfer- und Transkulturationsprozesse sogar zu einer Dekulturation, ${ }^{36}$ also zur „Verlernung“ der ursprünglichen eigenen Kultur geführt haben, soll hier nicht erörtert werden.

\subsection{Unvollständiger Spracherwerb?}

Insgesamt ergibt sich eingedenk der immer spärlicheren Inputsituation der Ungarndeutschen in den vergangenen Jahrzehnten das grundlegende Dilemma: Liegt bei diesen Phänomenen tatsächlich Erosion oder einfach unvollständiger Spracherwerb (incomplete acqusition, Montrul 2009) vor? Zu dessen Klärung bedarf es fundierter Langzeituntersuchungen.

\section{SCHLUSSBEMERKUNGEN}

Die durchgeführte datensensitive empirische Betrachtung hat, indem sie sich in einem ungarndeutschen Kontextrahmen mit dem informellen Alltagssprachgebrauch der genuinen Kommunikationsvarietät im nähesprachlichen Modus auseinandersetzte, eine besondere Kultur der diskursiven Mehrsprachigkeit unter Bedingungen intensiver und permanenter Sprachkontakte beschrieben. Der Sprachverwendungstyp ,Kontaktdeutsch ' gilt im gegebenen Kontext als die pragmatisch neutrale (,unmarkierte“) Sprachwahl. Dabei war zu berücksichtigen, dass mündlicher Sprachgebrauch zwischen einer gewissen Normorientierung und variablen pragmatischen Spielräumen oszilliert.

Es ist klar geworden, dass Sprache als komplexes adaptives System besondere Ausprägungen generieren kann: Die Studie förderte ein heterogenes Ensemble von kontaktinduzierten kommunikativen Mustern und Sprachformen sowie bilinguale Koproduktionen an der Schnittstelle zwischen mehreren Sprach(varietät)en mit einer Bandbreite vertikaler und horizontaler sowie inter- und intrasituativer Variation zutage; in vielen Belegen waren dabei Kontextualisierungshinweise zu beobachten. Angesichts der weitgehenden Fluktuation des Sprachgebrauchs besteht ein allgemeines Problem

35 Beispielsweise die Kontraktionen von Präposition und bestimmtem Artikel (am, beim etc., z. B. am freito:g, Nadasch, E_0142, S. 11) und die mehr oder weniger festen Wortgruppen (in die schul; siehe Beleg 2), vgl. Riehl (2015: 283 und 287).

Zum Begriff vgl. Lüsebrink (2016: 144). 
bei den Daten zu Deutsch als Minderheitensprache darin, zu eruieren, was ist konstant, was ist kollektiv - und was ist einmalig und individuell?

In der Mehrsprachigkeitsliteratur wird im Falle von Kontaktphänomenen sprecherseits meist systematische Intentionalität vorausgesetzt und dementsprechend sind Interpretationsbemühungen im Hinblick auf die Sprachwahl und/oder auf den Einsatz bestimmter Redemittel charakteristisch. Aus den Beobachtungsdaten des Projekts lässt sich jedoch schließen, dass oft keine ausgeprägte konversationelle Funktion für den Gebrauch der einen oder anderen Sprache oder Sprachform feststellbar ist. Diesen Befund stützt auch die Bemerkung von Berend (2013: 109), derzufolge ,"motivlose Variation" auch im deutschen Sprachraum häufig der Fall ist und dass auch einheimische [also bundesdeutsche - Anmerkung von mir: C. F.] Dialektsprecher häufig Varianten mischen, ohne dafür offensichtliche Gründe zu haben“".

Schließlich kristallisierten sich Konturen einer in gewisser Weise „postfränkischen“ Dialektausprägung, also einer spezifischen sprachlich-kommunikativen „GrenzgängerVarietät" heraus, wobei auch deutlich wurde, dass sich in der kommunikativen Welt von Ungarndeutschen zunehmend Erosionserscheinungen bemerkbar machen: Die Wortfluency lässt nach und eine langsame Restrukturierung des Systems setzt ein, die wohl in semantischen und morphologischen Domänen anfängt. Dies führt sukzessiv zu einer Abnahme der Funktionstüchtigkeit ,ungarndeutscher“ Dialektvarietäten.

\section{Literatur}

ADAMOU, Evangelia (2019) „Corpus linguistic methods.“ In: J. Darquennes/J. C. Salmons/W. Vandenbussche (Hrsg.), Language Contact. An International Handbook. Berlin/Boston: De Gruyter Mouton, 638-653.

ANSTATT, Tanja (2011) „Sprachattrition. Abbau der Erstsprache bei russisch-deutschen Jugendlichen." Wiener Slawistischer Almanach 67, 7-31.

AUER, Peter (1999) „From code-switching via language mixing to fused lects: toward a dynamic typology of bilingual speech." International Journal of Bilingualism, 3, 309-332.

AUER, Peter (2009) „Competence in performance: Code-switching und andere Formen bilingualen Sprechens.“ In: I. Gogolin/U. Neumann (Hrsg.), Streitfall Zweisprachigkeit. The Bilingualism Controversy. Wiesbaden: VS Verlag für Sozialwissenschaften, 91-110.

BEREND, Nina (2013) „Varietätenwandel im Kontakt. Die Entwicklung des Sprachgebrauchs deutschsprachiger Minderheiten im bundesdeutschen Kontext." In: A. Deppermann (Hrsg.), Das Deutsch der Migranten. Berlin/Boston: De Gruyter, 81-112.

DE BOT, Kees (2018) „Dynamische Modellierung von Spracherwerb. Sprache als dynamisches System." In: J. Roche/E. Terrasi-Haufe (Hrsg.), Mehrsprachigkeit und Spracherwerb. Tübingen: Narr Francke Attempto, 142-150.

DE BOT, Kees/Wander LOWIE/Marjolijn VERSPOOR (2007) „A Dynamic Systems Theory approach to second language acquisition." Bilingualism: Language and Cognition 10, 7-21. 
DE BOT, Kees/Bert WELTENS (1991) „Recapitulation, regression, and language loss.“ In: H. W. Seliger/R. M. Vago (Hrsg.), First Language Attrition. Cambridge: Cambridge University Press, 31-51.

DORIAN, Nancy C. (2009) „Sociolinguistic dimensions of language death.“ In: N. Coupland/A. Jaworski (Hrsg.), The New Sociolinguistics Reader. Basingstoke: Palgrave Macmillan, 541-559.

DUDENREDAKTION/SCHOLZE-STUBENRECHT, Werner (Hrsg.) (1999) Duden. Das große Wörterbuch der deutschen Sprache in zehn Bänden. 3., völlig neu bearb. u. erw. Aufl. Mannheim/Leipzig/Wien/Zürich: Dudenverlag.

ELLER-WILDFEUER, Nicole (2013) „Deutschböhmische Minderheitensprachen.“ In: G. Koch (Hrsg.), Sprachminderheit, Identität und Sprachbiographie. Regensburg: Edition Vulpes, 85-108.

FÖLDES, Csaba (2005) Kontaktdeutsch: Zur Theorie eines Varietätentyps unter transkulturellen Bedingungen von Mehrsprachigkeit. Tübingen: Narr.

FÖLDES, Csaba (2013) „Sprachliche Praktiken im Spannungsfeld von Variation und Mehrsprachigkeit: Ein Beitrag zur Empirie.“ In: K. Schneider-Wiejowski/B. Kellermeier-Rehbein/J. Haselhuber (Hrsg.), Vielfalt, Variation und Stellung der deutschen Sprache. Berlin/Boston: De Gruyter, 119-142.

FÖLDES, Csaba (2016a) „Diskurse im Wirkungsraum von Zweisprachigkeit. Werkstattbericht aus einem Forschungs- und Dokumentationsprojekt." In: M. Duś/R. Kołodziej/T. Rojek (Hrsg.), Wort-Text-Diskurs. Frankfurt a. Main: Peter Lang, 321-336.

FÖLDES, Csaba (2016b) „Ungarndeutsche Sprachvariation und Mehrsprachigkeit. Ein Korpusprojekt auf der Basis von empirischer Feldforschung und Online-Sprachdokumentation." Sprachtheorie und germanistische Linguistik 26/2, 167-190.

FOSCHI ALBERT, Marina (2018) „Eine linguistische Annäherung an das Thema Sprachverfall. Mit Berücksichtigung der Frage, ob Sprachwandel Gegenstand des DaF-Unterrichts sein soll.“ Glottodidactica 45, 105-118.

GARDNER-CHLOROS, Penelope (2009) Code-switching. Cambridge: Cambridge University Press.

GEHL, Hans (2005) Wörterbuch der donauschwäbischen Lebensformen. Stuttgart: Steiner. GRIMM, Jacob und Wilhelm (1984) [Erstausg. 1854] Deutsches Wörterbuch. 1. München: Deutscher Taschenbuch Verlag.

GRIMM, Jacob und Wilhelm (1991) [Erstausg. 1905] Deutsches Wörterbuch. 16. München: Deutscher Taschenbuch Verlag.

HAMERS, Joasiane F./Michel H. A. BLANC (2005) Bilinguality and Bilingualism. Second Edition. Cambridge: Cambridge University Press.

HUNEKE, Hans-Werner/Wolfgang STEINIG (2013) Deutsch als Fremdsprache. Eine Einführung. 6., neu bearb. u. erw. Aufl. Berlin: Schmidt.

KNIPF-KOMLÓSI, Elisabeth (2011) Wandel im Wortschatz der Minderheitensprache. Am Beispiel des Deutschen in Ungarn. Stuttgart: Steiner.

KÖPKE, Barbara (2019) „First language Attrition. From bilingual to monolingual proficiency?" In: A. De Houwer/L. Ortega (Eds.), The Cambridge Handbook of Bilingualism. Cambridge: Cambridge University Press, 349-365. 
LÜSEBRINK, Hans-Jürgen (2016) Interkulturelle Kommunikation: Interaktion, Fremdwahrnehmung, Kulturtransfer. 4., akt. und erw. Aufl. Stuttgart: Metzler.

MATRAS, Yaron (2009) Language Contact. Cambridge/New York: CUP.

MONTRUL, Silvina A. (2009) Incomplete acquisition in bilingualism. Re-examing the age factor. Amsterdam: Benjamins.

MÜLLER, Natascha (2017) Code-Switching. Tübingen: Narr Francke Attempto.

PARADIS, Michel (2004) A Neurolinguistic Theory of Bilingualism. Amsterdam: Benjamins.

PAUWELS, Anne (1986) „Diglossia, immigrant dialects and language Maintenance in Australia.“ Journal of Multilingual and Multicultural Development 7, 13-30.

RIEHL, Claudia (2014) Mehrsprachigkeit. Eine Einführung. Darmstadt: Wissenschaftliche Buchgesellschaft.

RIEHL, Claudia (2015) „Language attrition, language contact and the concept of relic variety: the case of Barossa German." International Journal of the Sociology of Language 236, 261-293.

RIEHL, Claudia (2016) „Reliktvarietät, Herkunftssprache, Minderheitensprache und neue Mehrsprachigkeit: Das Barossa-Deutsche als Beispiel für die Dynamik der deutschen Sprache in Übersee.“ In: A. N. Lenz (Hrsg.), German Abroad. Perspektiven der Variationslinguistik, Sprachkontakt- und Mehrsprachigkeitsforschung. Göttingen: V \& R Unipress/Vienna University Press, 241-267.

ROTH, Kersten Sven (2015) Diskursrealisationen: Grundlegung und methodischer Umriss einer pragmatisch-interaktionalen Diskurssemantik. Berlin: Erich Schmidt.

SCHMID, Hans-Jörg (2007) „Entrenchment, salience, and basic levels.“ In: D. Geeraerts/H. Cuyckens (Eds.), The Oxford Handbook of Cognitive Linguistics. New York/Oxford University Press, 117-138.

SCHMID, Monika (2011) Language Attrition. Cambridge: Cambridge University Press.

SILVA-CORVALÁN, Carmen (1983) „Code-Shifting patterns in Chicano Spanish.“ In: L. Elías-Olivares (Hrsg.), Spanish in the U.S. Setting. Beyond the Southwest. Rosslyn, VA: National Clearinghouse for Bilingual Education, 69-87.

SZABÓ, Csilla Anna (2010) Language shift und Code-mixing. Deutsch-ungarischrumänischer Sprachkontakt in einer dörflichen Gemeinde in Nordwestrumänien. Frankfurt a. Main: Peter Lang.

TABOURET-KELLER, Andrée (1969) „Plurilinguisme et interférences.“ In: A. Martinet (dir., avec la collab. de Jeanne Martinet et Henriette Walter), La linguistique. Guide alphabétique. Paris: Éditions Denoël, 305-310.

WILDFEUER, Alfred (2017) Sprachkontakt, Mehrsprachigkeit und Sprachverlust. Deutschböhmisch-bairische Minderheitensprachen in den USA und Neuseeland. Berlin/Boston: De Gruyter.

ZIEM, Alexander/Alexander LASCH (2013) Konstruktionsgrammatik. Konzepte und Grundlagen gebrauchsbasierter Ansätze. Berlin/Boston: De Gruyter. 


\title{
Zusammenfassung \\ BILINGUAL GEPRÄGTE KOMMUNIKATIONSSTRUKTUREN \\ AUF DER GRUNDLAGE FRÄNKISCHBASIERTER \\ UNGARNDEUTSCHER MUNDARTEN DER GEGENWART
}

Die vorliegende evidenzbasierte Studie stellt auf Basis eines Forschungs- und Dokumentationsprojekts aktuelle ungarndeutsche Kontaktvarietäten fränkischer Provenienz aus Südungarn vor und erschließt charakteristische Prozesse, Muster und Resultate intensiven deutsch-ungarischen Kontakt- bzw. Interaktionsgeschehens. Es werden u. a. spezifische Zwischenformen und Verbindungen aus den verfügbaren Kodes der Sprecher aufgedeckt: Mittels der Beschreibung zahlreicher overter und coverter Transferenzprozesse, Kode-Umschaltungen, bilingualer Dopplungen etc. konnte das Profil einer gleichsam ,postfränkischen“ Dialektausprägung, also einer spezifischen sprachlich-kommunikativen „Grenzgänger-Varietät“, herausgearbeitet werden. Es wurde klar, dass bei den ermittelten Kontaktphänomenen prototypisch drei Klassen eine Rolle spielen: (a) interlinguale Transfers/Übernahmen, (b) zwischensprachliche Kopien und (c) Sprachalternierungen. Die ersten beiden Manifestationsarten fasst der Aufsatz unter Hybridität zusammen, während Typ (c) als Synkretismus betrachtet wird. Dabei wurde auch deutlich, dass sich in der kommunikativen Welt von Ungarndeutschen zunehmend Erosionserscheinungen zeigen: Die Wortfluency lässt nach und eine langsame Restrukturierung des Systems setzt ein, die augenscheinlich in den semantischen und morphologischen Domänen anfängt. Dies führt sukzessiv zu einer Abnahme der Funktionsvitalität ungarndeutscher Dialektvarietäten.

Schlüsselwörter: Mehrsprachigkeit, Sprachkontakt, Interkulturalität, deutsche Dialekte

\author{
Abstract \\ BILINGUAL COMMUNICATIVE STRUCTURES \\ BASED ON RECENT FRANCONIAN-BASED \\ GERMAN DIALECTS IN HUNGARY
}

This evidence-based study presents current German contact varieties of Franconian origin from Southern Hungary. Based on a research and documentation project, the paper reveals characteristic processes, patterns and results of intensive German-Hungarian contact and interaction. Among other things, the research has revealed specific intermediate forms and convergences that could be gathered from the speakers' language use. By describing numerous overt and covert transfer processes, code switches, bilingual doublings and similar phenomena, the profile of a "post-Franconian" dialect manifestation as a specific linguistic and communicative "cross-border variety" was developed. Within the detected contact phenomena, three prototypical classes could be identified: (a) interlingual transfers/adoptions, (b) instances of interlingual copying, and (c) instances of code-switching. The paper presents the first two types of phenomena as 
hybridisms, while type (c) is regarded as syncretism. It also became clear that the language of the Germans in Hungary is increasingly affected by phenomena of erosion. Word fluency is diminishing and a slow restructuring of the system is beginning to take place, probably first in the semantic and morphological domains. At a second stage, this can lead to a decrease in the functional vitality of German dialect varieties in Hungary.

Keywords: multilingualism, language contact, interculturality, German dialects

\section{Povzetek \\ DVOJEZIČNI VIDIKI KOMUNIKACIJSKIH STRUKTUR V SODOBNIH FRANKOVSKONEMŠKIH NAREČNIH GOVORIH NA MADŽARSKEM}

Pričujoča empirično zasnovana študija predstavlja aktualne madžarskonemške stične jezikovne zvrsti frankovskonarečnega izvora na južnem Madžarskem. Izvedena je bila v okviru raziskovalnega in dokumentacijsko zasnovanega projekta, v okviru katerega so bili raziskovani karakteristični procesi, vzorci in rezultati intenzivnih nemško-madžarskih stikov in interakcij.

Študija med drugim razkriva specifične vmesne oblike in povezave iz dostopnih jezikovnih kodov govorcev. S pomočjo opisa številnih očitnih in zakritih transferenčnih procesov, menjavanja kodov, dvojezičnih podvajanj itd. je bilo mogoče opisati profil „postfrankovskega“" narečja, torej specifične „vmesne“ jezikovnosporazumevalne zvrsti. Raziskava je jasno pokazala, da imajo pri tem prototipično vlogo tri vrste jezikovnostičnih pojavov: (a) medjezikovni trastferji/prevzemanja, (b) medjezikovne kopije in (c) medjezikovno preklapljanje. Prvi dve vrsti pojavov sta v članku poimenovani kot hibridnost, tip (c) pa je označen s pojmom sinkretizem. Pri raziskovanju se je jasno pokazalo tudi, da se v madžarski nemščini čedalje bolj pojavlja erozija. Tekoči govor (Wortfluency) je začel usihati, pojavljati se je začelo prestrukturiranje sistema, ki se evidentno začenja $\mathrm{v}$ semantičnih in morfoloških segmentih, to pa sukcesivno vodi $\mathrm{k}$ zmanjšanju funkcionalne vitalnosti madžarskonemških narečnih zvrsti.

Ključne besede: večjezičnost, jezikovni stik, medkulturnost, nemška narečja 